Research Paper

\title{
Human colorectal cancer progression correlates with LOX-induced ECM stiffening
}

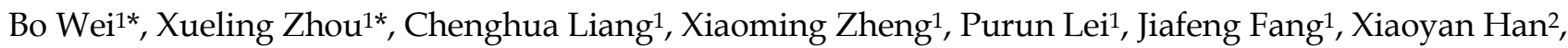
Lijing Wang ${ }^{3}$, Cuiling $\mathrm{Qi}^{\mathrm{B}}$, Hongbo Wei ${ }^{1 凶}$

1. Department of Gastrointestinal Surgery, the Third Affiliated Hospital, Sun Yat-sen University, Guangzhou, Guangdong 510630, China;

2. Central Laboratory, the Third Affiliated Hospital, Sun Yat-sen University, Guangzhou, Guangdong 510630, China;

3. Vascular Biology Research Institute, School of Basic Course, Guangdong Pharmaceutical University, Guangzhou, Guangdong 510006, China.

* These authors contributed equally to this work.

$\triangle$ Corresponding authors: Cuiling Qi or Hongbo Wei. Address: Vascular Biology Research Institute, School of Basic Course, Guangdong Pharmaceutical University, Guangzhou Higher Education Mega Center, Guangzhou, Guangdong 510006, China, or Department of Gastrointestinal Surgery, the Third Affiliated Hospital of Sun Yat-sen University, Guangzhou, Guangdong 510630, China; Tel: +86-020-39352397; E-mail: qicuiling12345@163.com or drweihb@126.com.

(C) Ivyspring International Publisher. This is an open access article distributed under the terms of the Creative Commons Attribution (CC BY-NC) license (https://creativecommons.org/licenses/by-nc/4.0/). See http://ivyspring.com/terms for full terms and conditions.

Received: 2017.05.29; Accepted: 2017.10.08; Published: 2017.11.01

\begin{abstract}
Some solid tumors are characterized by extracellular matrix (ECM) remodeling and stiffening, which is related to solid tumor progression and aggression. However, the relationship between ECM stiffness and colorectal cancer (CRC) remains unclear. In this study, we investigated the relevance of ECM stiffness to clinicopathologic features using human CRC tissue microarrays. The results demonstrate that the expression of ECM components in CRC tissues is closely correlated with $C R C$ progression and poor prognosis, which indicates that ECM stiffness may be associated with CRC development. We further studied lysyl oxidase (LOX) expression in CRC tissue and demonstrated that LOX expression is closely correlated with CRC progression. Previous studies showed that P-selectin-mediated platelet accumulation in CRC tissue may up-regulate LOX expression. Our findings indicate that P-selectin-mediated platelet aggregation may up-regulate LOX expression and enhance the remodeling and stiffening of the tumor ECM, which may promote the progression of colorectal cancer. Therefore, LOX may be a potential effective therapeutic target to treat colorectal cancer.
\end{abstract}

Key words: tissue stiffness, LOX, colorectal cancer.

\section{Introduction}

Colorectal cancer (CRC) is the third leading cause of cancer deaths, with a 5-year overall survival rate of $53.8-65.2 \%$ worldwide despite advances in diagnostic and therapeutic measures in recent years $[1,2]$. In the United States, CRC is the third most common cancer and the fourth most common cause of cancer death, with an average life risk of approximately $5 \%[3,4]$. In China, CRC is the fifth most common cancer and the fifth most common cause of cancer death. In another words, an estimated 376,300 patients were diagnosed with colorectal cancer, and an estimated 191,000 deaths were attributable to colorectal cancer in 2015 [5], with an obvious increase compared to the statistical data from 2012 [6].

Research has shown that some solid tumors are stiffer than their surrounding tissues, and tumor stiffness is related to solid tumor progression and aggression $[7,8]$. The stiffness is contingent on tumor extracellular matrix $(\mathrm{ECM})$, which has been increasingly recognized as more than just a minor player in the constitution, development and regulation of the tumor microenvironment [9]. The enhancement of tumor ECM stiffness is characterized by collagen deposition and remodeling, especially manifested by the cross-linking of collagen proteins $[10,11]$. 
In the process of malignant transformation, progression and metastasis of tumors, the ECM is modified by many enzymes, including matrix metalloproteinases (MMPs) and lysyl oxidase (LOX) family oxidases. Importantly, LOX family oxidases (LOX and its family members, LOX-like proteins 1-4) are abnormally expressed in tumors and act as modifiers of the mechanical properties in the tumor microenvironment [12]. LOX is a secreted, copper-dependent amine oxidase and collagen cross-linker [13]. Our preliminary study showed that up-regulation of LOX expression can increase tumor stiffness, thereby promoting insulinoma growth in Rip1-Tag2 mice and that the LOX inhibitor, $\beta$-aminopropionitrile (BAPN), could inhibit tumor progression through decreasing the amount of collagen cross-links in tumors [14].

However, the relationship between ECM stiffness and colorectal cancer remains unknown. In this study, we investigated the relevance of ECM stiffness to clinicopathologic features using human colorectal cancer tissue microarrays. We found that ECM stiffness in colorectal cancer tissues was closely correlated to CRC progression and to a poor prognosis. Our previous finding also indicated that many platelets aggregate around colorectal tumor cells, and platelet accumulation was closely related to colorectal cancer progression. Moreover, P-selectinmediated platelet accumulation promoted intestinal tumorigenesis in $\mathrm{Apc}^{\mathrm{Min} /+}$ mice [15]. Our studies also demonstrated that LOX expression is correlated with CRC progression and poor prognosis. Thus, our findings indicate that P-selectin-mediated platelet aggregation may up-regulate LOX expression and enhance the remodeling and stiffening of the tumor ECM, which promotes the progression of colorectal cancer.

\section{Materials and Methods}

\section{Human colorectal cancer tissue samples}

Human colorectal cancer (CRC) tissue microarrays were obtained from Alenabio ( $\mathrm{Xi}^{\prime}$ an, Shanxi, China). The tumor tissues were obtained from CRC patients who were in different stages classified by expert pathologists according to the TNM Staging 7th Edition of the American Joint Committee on Cancer (AJCC). One tissue microarray, including 48 colorectal cancer tissue samples with prognostic information, was used for a correlation analysis between tissue stiffness and prognosis. Another tissue microarray, including 207 colorectal cancer tissue samples, was used for the mechanism research on tissue stiffening in CRC.

\section{Histopathological staining}

Human colorectal cancer tissue microarrays were stained with H\&E, Masson's trichrome and reticulin by using reagents and kits from Maxim-Bio (Fuzhou, Fujian, China). For histopathological staining quantitation, randomly chosen fields were examined using a $40 \times$ objective lens. The images were analyzed to quantify the collagen, collagenous fiber, and reticulin expression using Image-Pro Plus image analysis software (Image-Pro-Plus, version 6.0, Media Cybernetics) under a $400 \times$ objective field. The images were evaluated by two experimenters.

\section{Immunohistochemical staining}

For immunohistochemical staining, 4- $\mu \mathrm{m}$ sections of the human colorectal cancer tissue arrays were used. Briefly, the slides were subsequently dewaxed, rehydrated, and incubated in $3 \%$ peroxide-methanol at $37^{\circ} \mathrm{C}$ for 30 minutes to quench endogenous peroxidase. Next, the sections were treated with $10 \%$ bovine serum albumin (Sigma-Aldrich, St Louis, MO, USA) to block the non-specific binding and incubated with anti-collagen type I antibody (1:100 dilution, Abcam, Cambridge, CB, UK) or anti-LOX antibody (1:100 dilution, Abcam) overnight at $4{ }^{\circ} \mathrm{C}$. The slides were then incubated with a horseradish peroxidase-conjugated goat anti-rabbit IgG antibody (ZSGB-BIO, Beijing, China) at $37^{\circ} \mathrm{C}$ for 50 minutes. All the sections were stained with diaminobenzidine solution (DAB, Dako Cytomation, Hamburg, Germany) and counterstained with hematoxylin. The images were analyzed to quantify the LOX expression using IPP (version 6.0) under a $400 \times$ objective field. The images were evaluated by two experimenters.

\section{Evaluation of immunohistochemical staining}

The tissue sections of immunohistochemical staining for collagen type I were scored as previously described [16]. Briefly, the evaluation of staining was performed by separately by two pathologists blinded to the clinical parameters. The staining intensity scores of collagen type I were 0 (negative), 1 (weak), 2 (medium), and 3 (strong). The staining extent scores were $0(0 \%), 1(1-25 \%), 2$ (26-50\%), $3(51-75 \%)$, and 4 $(76-100 \%)$ according to the percentages of the positive staining areas. The final staining score (0-7) for collagen type I was evaluated according to the staining intensity and staining extent. The final staining scores of $0-4$ and 5-7 were respectively regarded as low and high expression.

\section{Western blotting}

Colorectal cancer tissues from patients were grinded under liquid nitrogen condition. Proteins 
were extracted from cancer tissues in RIPA lysis buffer. Electrophoresis was performed under a $10 \%$ SDS polyacrylamide gel. Then proteins were transferred onto polyvinylidene fluoride membranes and incubated with a primary antibody against LOX (1:1000 dilution, Abcam), collagen type I (1:1000 dilution, Abcam) and GADPH (1:10000 dilution, Abcam). After that, the membranes were incubated with horseradish peroxide (HRP)-conjugated secondary goat anti-mouse (1:5000 dilution, BioRad) or goat anti-rabbit (1:5000 dilution, Abcam) antibody. Bands were visualized under $\mathrm{ChemiDoc}^{\mathrm{TM}}$ Touch Imaging System.

\section{Statistical analyses}

The SPSS software package (version 19, SPSS Inc, Chicago, IL) was used to analyze the data, and Sigmaplot software (version 10.0, Systat Software Inc, San Jose, CA) was used to draw the statistical charts. A Student's t-test was used to confirm the statistical significance. The Kaplan-Meier method was used to plot survival curves. And the Cox multivariate proportional hazards model was used to analyze the significance of survival variables. For all tests, $p<0.05$ was considered as statistical significance.

\section{Results}

Correlation analysis between collagen Type I expression and prognosis of colorectal cancer.

It has been reported that ECM stiffness is closely associated with tumor progression and prognosis in many types of tumors [10-12], but little is known about its association with colorectal cancer. Through immunohistochemical staining of CRC tissue microarrays, we first investigated the expression of collagen type I, the most important component of the ECM, and the correlation between collagen type I expression and prognosis of colorectal cancer was analyzed. The results showed that the overall survival rate of CRC patients with high-expression of collagen type I was lower than those with low-expression of collagen type I (Figure 1). That is, the content of collagen type I, or the ECM stiffness, was correlated with the prognosis of CRC patients.

\section{Collagen deposition increases in colorectal cancer progression}

Next, the correlation between collagen type I and the clinicopathologic features of the CRC patients, including the AJCC staging, WHO grading, T staging, and lymph node or distant organ metastasis, was analyzed. The results indicated that collagen type I expression was stronger in stage III/IV CRC tissues than in stage I/II tissues (Figure 2C). As for collagen type I expression across individual WHO grades (1-3), there was no difference in collagen type I expression between grade 1 and grade 2 . However, collagen type I expression was significantly increased in grade 3 colorectal cancer tissues compared with grade 2 tissues (Figure 2D). Collagen type I expression in CRC with metastasis (N1/M1) was stronger compared to those in the tissues with no lymph node metastasis (N0/ M0) (Figures 2E, 2F), and its expression in the stage T3/T4 CRC group was significantly increased compared to the stage T2 group (Figure 2G). The result was conformed by western blotting (Supplemental Figure 1). Thus, high collagen type I expression was correlated with worse clinicopathologic features of the CRC patients.

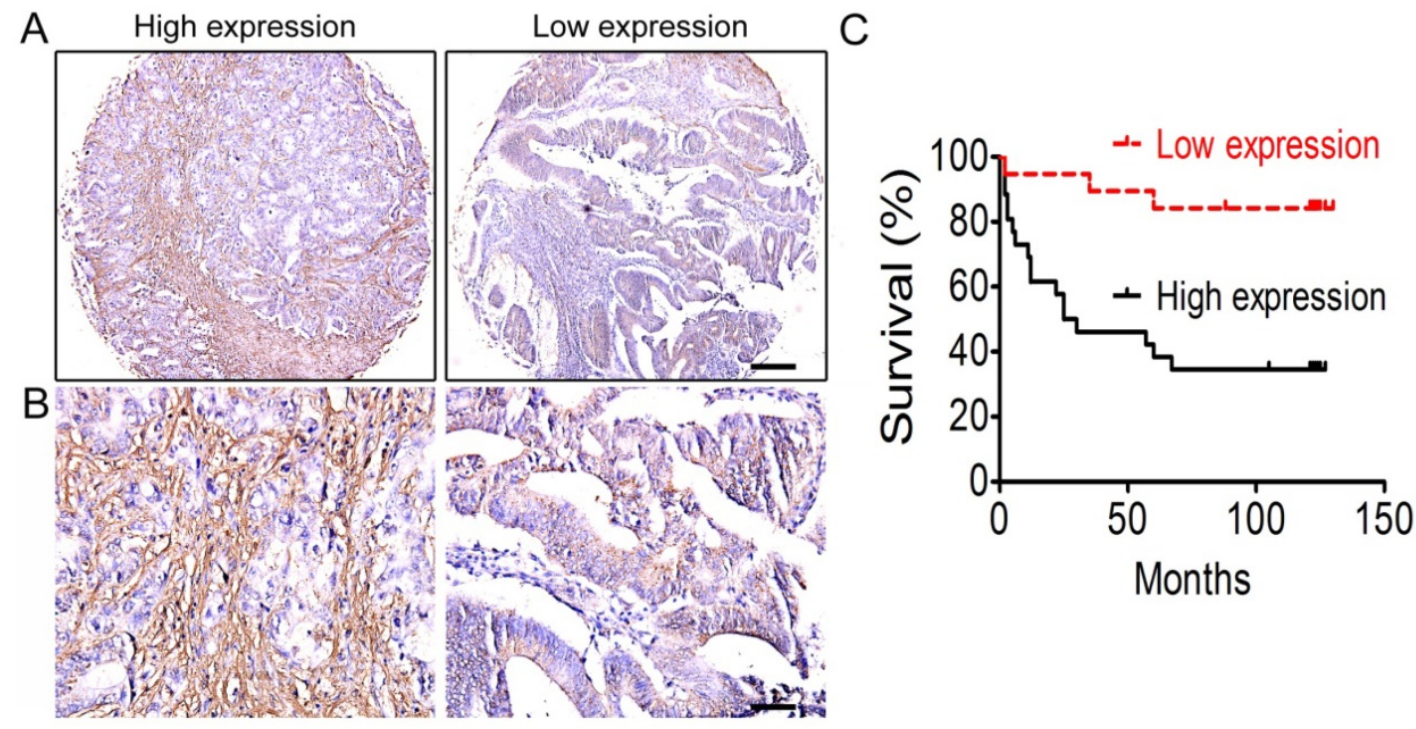

Figure 1. Correlation analysis between collagen type I expression and the prognosis of colorectal cancer. (A) Collagen type I immunohistochemical staining of colorectal cancer tissues under low power lens. Bar $=200 \mu \mathrm{m}$. (B) Collagen type I immunohistochemical staining of colorectal cancer tissues under high power lens. Bar $=50 \mu \mathrm{m}$. (C) The overall survival of the CRC patients between low-expression and high-expression of collagen type I. 


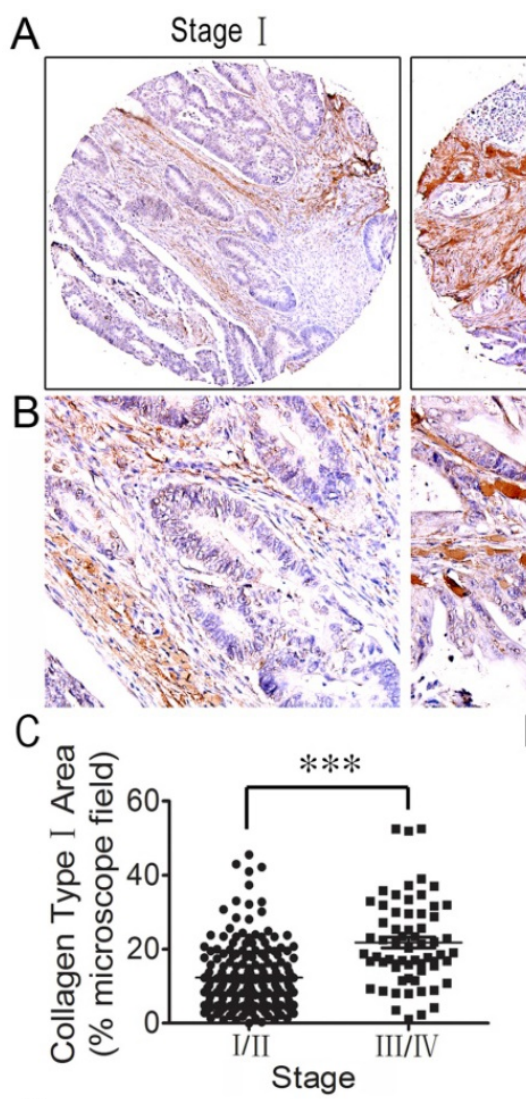

$\mathrm{F}$
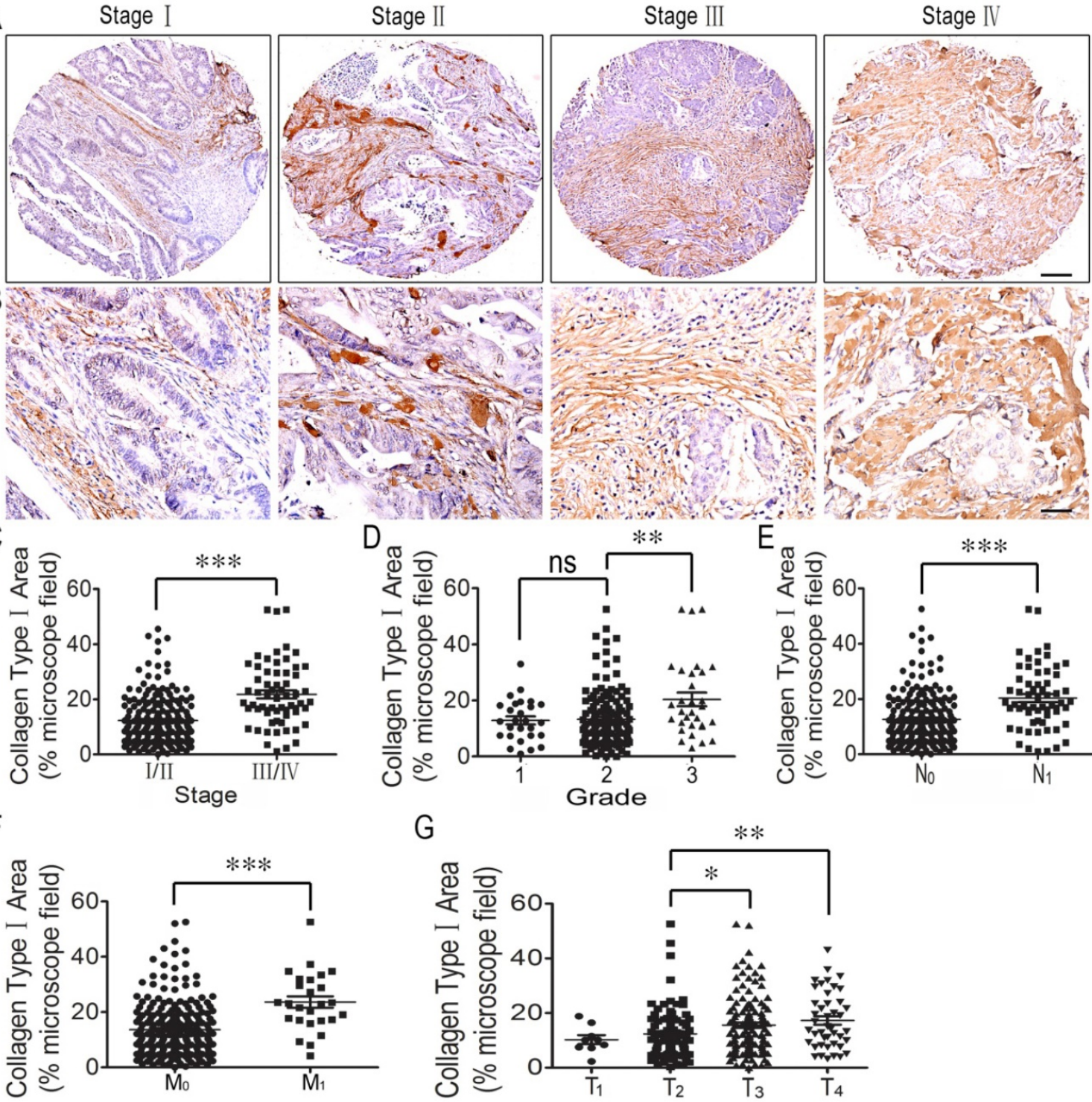

Figure 2. Correlation analysis between collagen type I expression and clinicopathologic features of colorectal cancer. (A) Collagen type I immunohistochemical staining of colorectal cancer tissues in different stages. Bar $=200 \mu \mathrm{m}$. (B) Collagen type I immunohistochemical staining of colorectal cancer tissues in different stages. Bar $=50 \mu \mathrm{m}$. (C) Collagen type I expression across individual AJCC-7th stages (I-IV). Collagen type I expression was stronger in stage III/IV CRC tissues than in stage I/II tissues. (D) Collagen type I expression across individual WHO grades (1-3). There was no difference in Collagen type I expression between grade 1 and grade 2. Collagen type I expression was significantly increased in grade 3 colorectal cancer tissues compared with grade 2 . (E) Collagen type I expression in CRC with lymph node metastasis $\left(\mathrm{N}_{\mathrm{I}}\right)$ was stronger compared to that in the tissues with no lymph node metastasis (No). ( $\mathrm{F}$ ) Collagen type I expression in CRC with lymph node metastasis $\left(M_{1}\right)$ was stronger compared to that in the tissues with no lymph node metastasis (Mo). (G) Collagen type I expression in the stage $\mathrm{T}_{3} / \mathrm{T}_{4} \mathrm{CRC}$ group was significantly increased compared to that in the stage $\mathrm{T}_{2}$ group. $*, p<0.05 ; * *, p<0.01 ; * * *, p<0.001$.

Collagen fiber content of the ECM was also studied using Masson's trichrome staining, and its correlation with the clinicopathologic features of CRC patients was also analyzed. The results showed that collagen fiber concentration was higher in stage III/IV CRC tissues than stage I/II tissues (Figure 3C), and the concentration increased from grade 1 tumors to grade 3 tumors (Figure 3D). Also, collagen fiber content in CRC with lymph node metastasis (N1) was significantly higher than in the tissues with no lymph node metastasis (N0) (Figure 3E). However, there was no difference in collagen fiber content in the different T stages (T1-T4) (Figure 3F).

\section{Correlation analysis between reticulin expression and clinicopathologic feature of colorectal cancer}

Reticulin is one type of albuminoid and an important component of the tumor ECM. Therefore, we investigated reticulin expression using reticulin staining and analyzed its correlation with the clinicopathologic features of CRC patients. The results showed that reticulin expression significantly increased in stage III/IV CRC tissues compared to stage I/II tissues (Figure 4A, B, C) and was stronger in grade $2 \mathrm{CRC}$ tissues than in grade 1 tissues, and even 
stronger in grade 3 tissues (Figure 4D). In addition, reticular fibers in CRC with lymph node metastasis (N1) were increased significantly compared to those with no lymph node metastasis (N0) (Figure 4E). However, there was no difference in reticulin expression in the different $\mathrm{T}$ stages (T1-T4) (Figure $4 \mathrm{~F})$.

\section{ECM stiffness of colorectal cancer increases through improving LOX expression}

The variations in the connective scaffold architecture induced by lysyl oxidase and metalloproteinase activity create different conditions of ECM density and stiffness [9]. Our previous report indicated that the up-regulation of LOX expression could increase tumor stiffness in spontaneous insuloma from Rip1-Tag2 mice; thus, we focused on this copper-dependent amine oxidase. The immunohistochemical staining and western blotting showed that LOX expression was up-regulated in CRC tissues and that the expression level was correlated with the progression of the tumor, including the tumor stage, lymph node metastasis and tumor grade (Figure 5 and Supplemental Figure $1)$.

\section{Discussion}

It is well known that human cancers exhibit intratumor heterogeneity [17]. The stroma is a considerable part of the tumor microenvironment, which includes vasculature, nerves, fibroblasts, infiltrated immune cells and the extracellular matrix components [9]. It is increasingly recognized that the ECM structure, composition and stiffness have profound effects on tissue development and pathologies of cancer [18]. Further, cell-ECM adhesion can profoundly modify cell shape and tissue organization and can dramatically regulate gene expression and cell behavior [19]. The importance of ECM remodeling to cancer is appreciated, but the relevance of stiffening is unclear [20]. In recent years, research has demonstrated that ECM stiffness is correlated with the progression and metastasis of many types of cancer [7, 8, 15]. However, the relationship between ECM stiffness and colorectal cancer remains unknown.
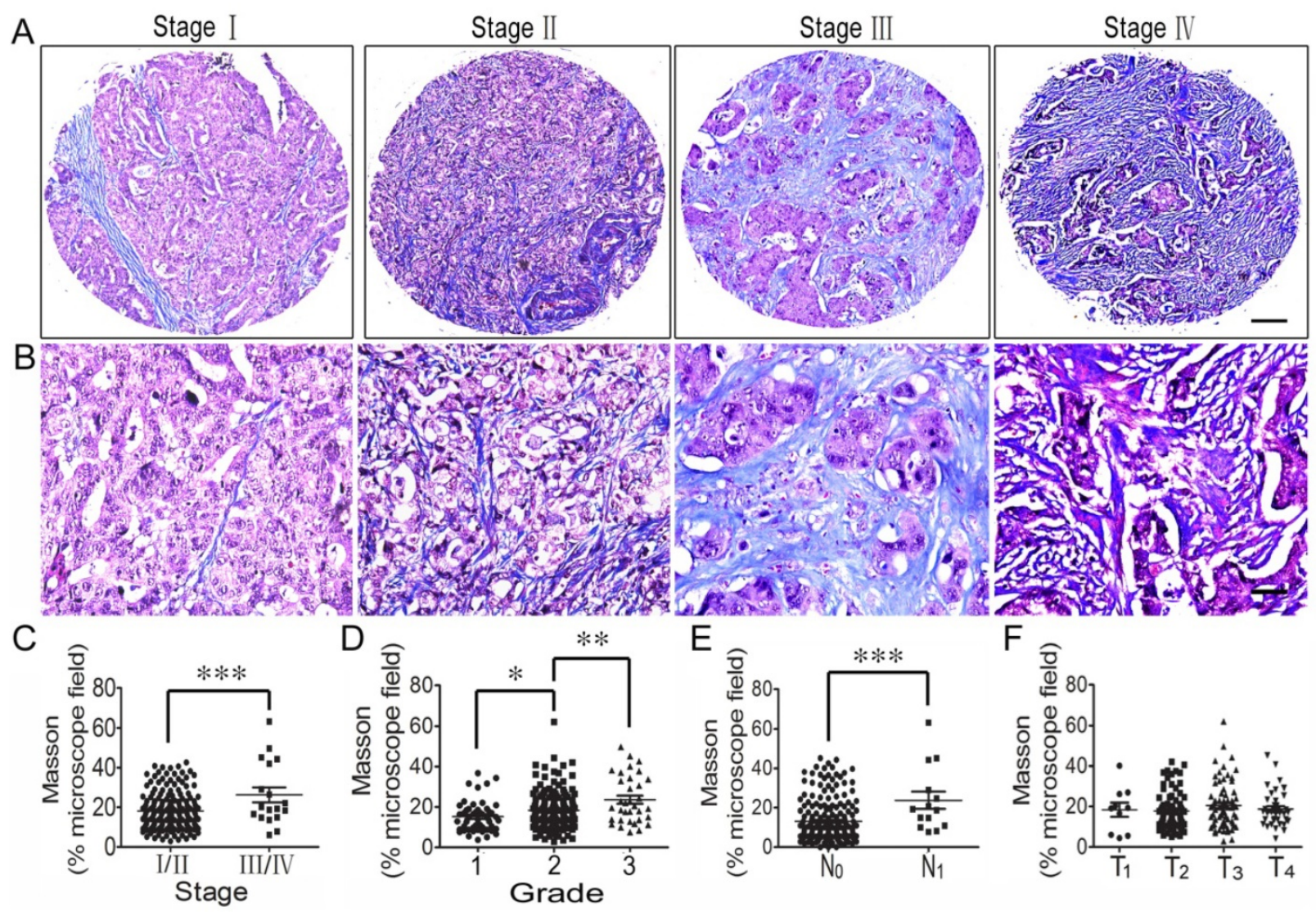

Figure 3. Correlation analysis between collagen fiber content and clinicopathologic features of colorectal cancer. (A) Masson trichrome staining of CRC tissues across individual AJCC-7th stages I-IV. Bar $=200 \mu \mathrm{m}$. (B) Masson staining of CRC tissues across individual AJCC-7th stages I-IV. Bar $=50 \mu \mathrm{m}$. (C) Elastin expression was significantly stronger in stage III/IV CRC tissues than in stage I/II tissues. (D) Collagen fiber content was higher in grade 2 CRC tissues than in grade 1 tissues and even stronger in grade 3 CRC tissues. (E) Collagen fiber content in CRC with lymph node metastasis $\left(N_{1}\right)$ was significantly stronger compared to that in the tissues with no lymph node metastasis $\left(\mathrm{N}_{0}\right)$. ( $(\mathrm{F})$ There was no difference in collagen fiber content in the different $\mathrm{T}$ stages $\left(\mathrm{T}_{1}-\mathrm{T}_{4}\right)$. $*, p<0.05 ; * *, p<0.01 ; * * *$, $p<0.001$. 

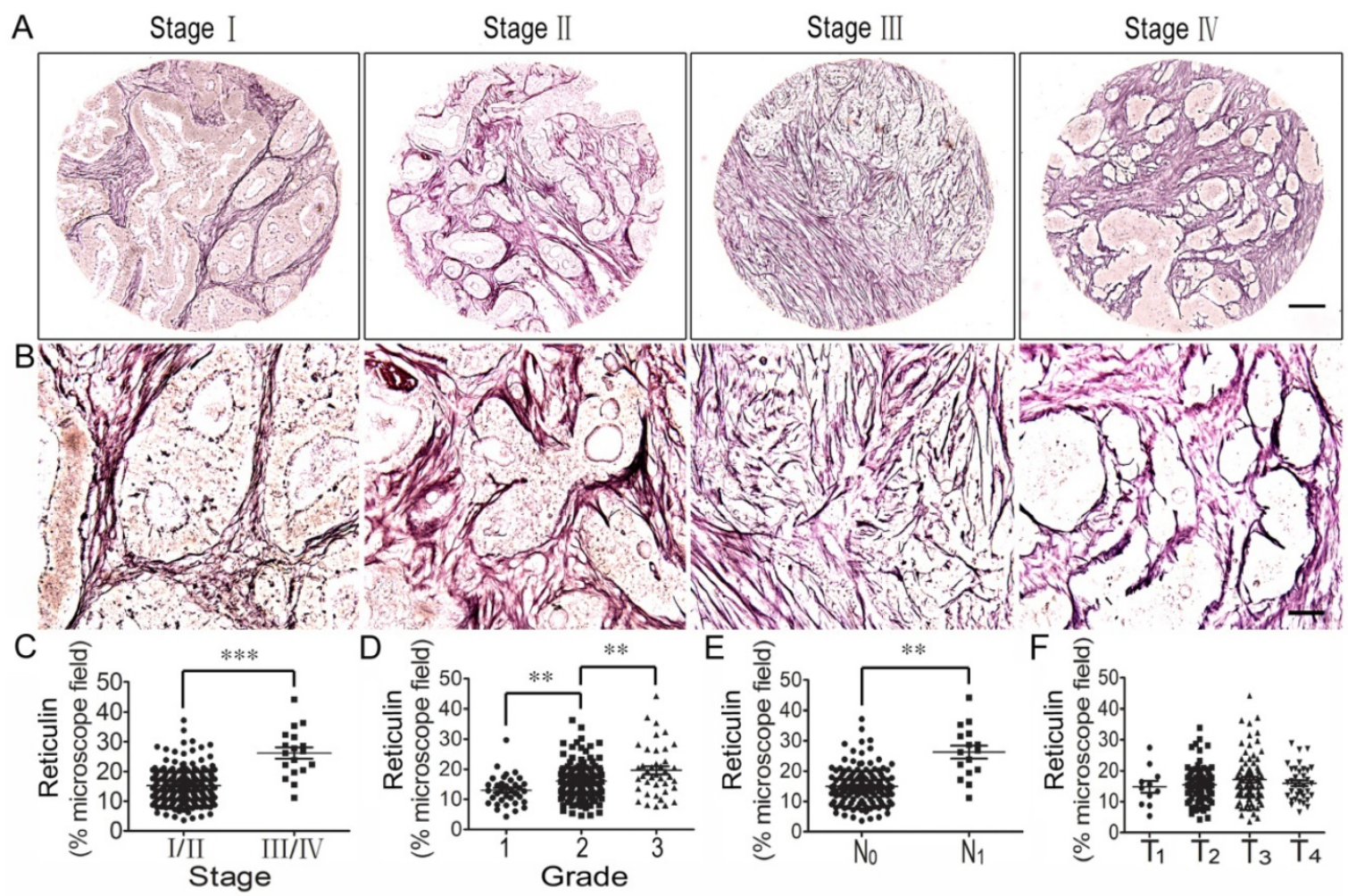

Figure 4. Correlation analysis between reticulin expression and clinicopathologic features of colorectal cancer. (A) Reticulin staining of $C R C$ tissues across individual AJCC-7th stages I-IV. Bar $=200 \mu \mathrm{m}$. (B) Reticulin staining of CRC tissues across individual AJCC-7th stages I-IV. Bar $=50 \mu \mathrm{m}$. (C) Reticulin expression was significantly stronger in stage III/IV CRC tissues than in stage I/II tissues. (D) Reticulin expression was stronger in grade 2 CRC tissues than in grade 1 tissues and even stronger in grade 3 tissues. (E) Reticulin expression in $C R C$ with lymph node metastasis $\left(N_{1}\right)$ was significantly stronger compared to that with no lymph node metastasis $\left(\mathrm{N}_{0}\right)$. ( $\left.\mathrm{F}\right)$ There was no difference in reticulin expression in the different $T$ stages $\left(\mathrm{T}_{1}-\mathrm{T}_{4}\right)$. **, $p<0.01 ; * * *, p<0.001$.
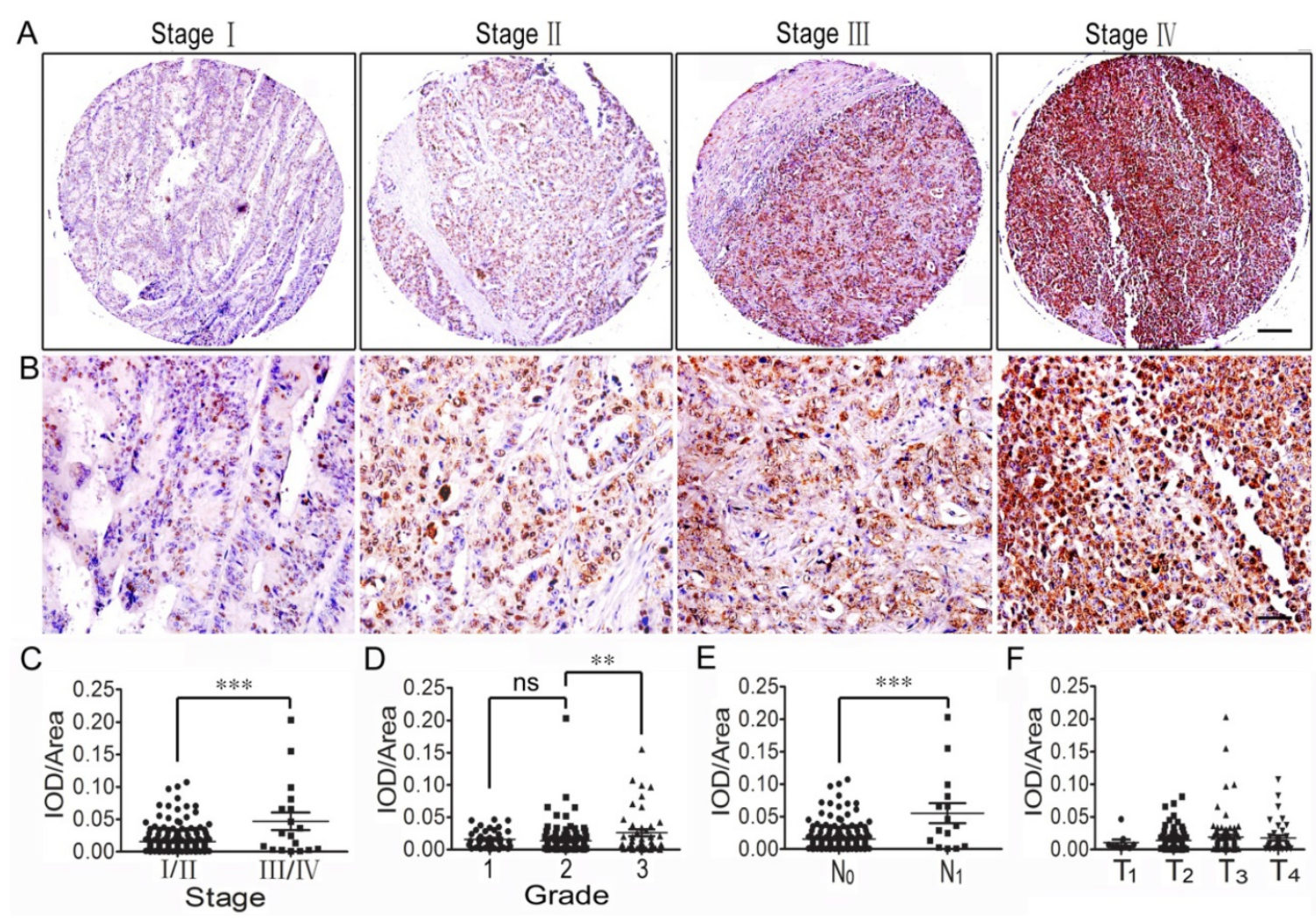

Figure 5. Correlation analysis between LOX expression and clinicopathologic features of colorectal cancer. (A) LOX expression of CRC tissues across individual AJCC-7th stages I-IV. Bar $=200 \mu \mathrm{m}$. (B) LOX expression of CRC tissues across individual AJCC-7th stages I-IV. Bar $=50 \mu \mathrm{m}$. (C) LOX expression was significantly stronger in stage III/IV CRC tissues than in stage I/II tissues. (D) LOX expression was stronger in grade 3 CRC tissues than grade 1 and 2 tissues, but no significant difference was found between grade 1 and 2 tissues. (E) LOX expression in CRC with lymph node metastasis $\left(N_{1}\right)$ was significantly stronger compared to that with no lymph node metastasis $\left(N_{0}\right)$. ( $\left.F\right)$ There was no difference in LOX expression in the different $T$ stages $\left(T_{1}-T_{4}\right)$. $* *, p<0.01 ; * * *, p<0.001$. 
In the current study, we first analyzed the relationship between the ECM stiffness of CRC tissues and the overall patient survival rate using human colorectal cancer tissue microarrays. The results indicate that a high expression of collagen type I correlate with a poor prognosis. We then investigated the relevance of ECM stiffness and clinicopathologic features and found that the collagen type I expression and collagen fiber deposition were higher in stage III/IV CRC tissues than in stage I/II tissues and increased from grade 1 tumors to grade 3 tumors. Reticulin expression also increased with the TNM stage progression and tumor grade elevation. These results indicated that ECM stiffness in colorectal cancer tissues was closely correlated with CRC progression and metastasis.

Research has shown that LOX is abnormally expressed in some tumors during malignant transformation, progression and metastasis. As a copper-dependent amine oxidase, LOX has collagen and elastin as its substrates and initiates the process of covalent intra- and intermolecular crosslinking of collagen and elastin by oxidatively deaminating specific lysine and hydroxylysine residues located in the telopeptide domains [21]. The covalent crosslinking and stabilization of these ECM structural components play a role in the homeostasis of connective tissue. Consequently, over-expression of these enzymes can increase tissue tension and ECM rigidity and may be involved in tissue fibrosis [13, 22]. Collagen crosslinking is induced predominantly by LOX and LOX-like (LOXLs) enzymes, which are synthesized by both stromal cells and cancer cells in the early stages of cancers in response to hypoxia [23, 24]. In this study, we investigated the relationship between LOX expression and the clinicopathologic features of CRC. The immunohistochemical staining showed that LOX expression was correlated with the progression of the tumor, including the tumor stage, $\mathrm{T}$ stage, tumor grade, and metastasis.

Our previous study indicated that LOX overexpression increases tumor stiffness in spontaneous insuloma of Rip1-Tag2 mice and that the tumor stiffening participates in the whole tumorigenesis [15]. The ECM stiffening is correlated with P-selectin-mediated platelet accumulation, and we found that P-selectin deficiency significantly decreased the tissue stiffness through the inhibition of LOX expression. Also, a LOX inhibitor, BAPN, can significantly abolish the collagen deposition and decrease the tumor stiffness, thereby inhibiting tumor growth [15]. The increased tumor ECM stiffness elicits diverse effects on tumor cell proliferation, differentiation, and migration and thus significantly modifies tumor progression [25-28]. As we previously reported, P-selectin-mediated platelet accumulation in colorectal cancer was also associated with tumor development [16, 29]. These results indicated that P-selectin-mediated platelet deposition might up-regulate LOX expression and then facilitate the covalent crosslinking of ECM components, enhance tumor ECM stiffness, and thereby promote colorectal cancer progression and invasion. Therefore, LOX may be a potential effective therapeutic target to treat human colorectal cancer.

\section{Supplementary Material}

Supplementary figure s1.

http://www.ijbs.com/v13p1450s1.pdf

\section{Acknowledgements}

This work was supported by grants from the National Science Foundation of China (81472825 to Bo Wei, 31500966 and 81773095 to Cuiling Qi), the Natural Science Foundation of Guangdong Province (2014A030313078 to Bo Wei), the Science and Technology Planning Project of Guangdong Province (2014A020212314 and 2015A020211029 to Cuiling Qi, 2013B021800078 to Xiaoyan Han, 2017A010103009 to Bo Wei, 2014B090901066 to Hongbo Wei), the Science and Technology Planning Project of Guangzhou City (201607010135 to Cuiling Qi, 2014Y2-00503 to Hongbo Wei), the Major Projects and Emerging Interdisciplinary Grant Program of Fundamental Research Funds for the Central Universities (16ykjc23 to Bo Wei).

\section{Competing Interests}

The authors have declared that no competing interest exists.

\section{References}

1. O'Connell JB, Maggard MA, Ko CY. Colon cancer survival rates with the new American Joint Committee on Cancer sixth edition staging. J Natl Cancer Inst. 2004; 96(19):1420-25.

2. Berrino F, De Angelis R, Sant M, Rosso S, Bielska-Lasota M, Coebergh JW, Santaquilani M. Survival for eight major cancers and all cancers combined for European adults diagnosed in 1995-99: results of the EUROCARE-4 study. Lancet Oncol. 2007;8(9):773-83.

3. Siegel RL, Miller KD, Jemal A. Cancer statistics, 2017. CA Cancer J Clin. 2017;67(1):7-30.

4. Eheman C, Henley SJ, Barbash RB, Jacobs EJ, Schymura MJ, Noone AM, Pan L, Anderson RN, Fulton JE, Kohler BA, Jemal A, Ward E, Plescia M, Ries LA, Edwards BK. Annual Report to the Nation on the Status of Cancer, 1975-2008. Cancer. 2012;118(9):2338-66.

5. Chen W, Zheng R, Baade PD, Zhang S, Zeng H, Bray F, Jemal A, Yu XQ, He J. Cancer statistics in China, 2015. CA Cancer J Clin. 2016;66(2):115-132.

6. Chen $\mathrm{W}$, Zheng $\mathrm{R}$, Zuo $\mathrm{T}$, Zeng $\mathrm{H}$, Zhang $\mathrm{S}$, He J. National cancer incidence and mortality in China, 2012. Chin J Cancer Res. 2016;28(1):1-11.

7. Samani A, Bishop J, Luginbuhl C, Plewes DB. Measuring the elastic modulus of ex vivo small tissue samples. Phys Med Biol. 2003;48(4):2183-98.

8. Samani A, Zubovits J, Plewes D. Elastic moduli of normal and pathological human breast tissues: an inversion-technique-based investigation of 169 samples. Phys Med Biol. 2007;52(6):1565-76.

9. Vannucci L. Stroma as an active player in the development of the tumor microenvironment. Cancer Microenvironment. 2015;8(3):159-66.

10. Acerbi I, Cassereau L, Dean I, Shi Q, Au A, Park C, Chen YY, Liphardt J, Hwang ES, Weaver VM. Human breast cancer invasion and aggression correlates with ECM stiffening and immune cell infiltration. Integr Biol (Camb). 2015;7(10):1120-34. 
11. Butcher DT, Alliston T, Weaver VM. A tense situation: forcing tumour progression. Nat Rev Cancer. 2009; 9:108-122.

12. Xiao Q, Ge G. Lysyl oxidase, extracellular matrix remodelingand cancer metastasis. Cancer Microenviron. 2012;5: 261-273.

13. Yamauchi $M$, Sricholpech $M$. Lysine post-translationalmodifications of collagen. Essays Biochem. 2012; 52:113-133.

14. Qi CL, Li JL, Guo SM, Li M, Li Y, Li J, Zhang Q, Zheng L, He X, Zheng X, He Y, Wang L, Wei B. P-selectin-mediated LOX expression promotes insulinoma growth in Rip1-Tag2 mice by increasing tissue stiffness. Int J Biol Sci. 2016;12(11):1289-97.

15. Qi CL, Li B, Guo SM, Wei B, Shao C, Li J, Yang Y, Zhang Q, Li J, He X, Wang L, Zhang Y. P-Selectin-mediated adhesion between platelets and tumor cells promotes intestinal tumorigenesis in $\mathrm{Apc}^{\mathrm{Min} /+}$ mice. Int J Biol Sci. 2015;11(6): 679-87.

16. Huang R, Huang D, Dai W, Yang F. Overexpression of HMGA1 correlates with the malignant status and prognosis of breast cancer. Mol Cell Biochem. 2015;404(1-2):251-7.

17. Michor F, Weaver VM. Understanding tissue context influences on intratumour heterogeneity. Nat Cell Biol. 2014;16(4):301-02.

18. Cassereaua L, Miroshnikova YA, Ou G, Lakins J, Weaver VM. A 3D tension bioreactor platform to study the interplay between ECM stiffness and tumor phenotype. J Biotechnol. 2015; 193:66-69.

19. McAllister SS, Weinberg RA. Tumor-host interactions: a far-reaching relationship. J Clin Oncol. 2010;28(26):4022-28.

20. Levental KR, Yu HM, Kass L, Lakins JN, Egeblad M, Erler JT, Fong SF, Csiszar K, Giaccia A, Weninger W, Yamauchi M, Gasser DL, Weaver VM. Matrix crosslinking forces tumor progression by enhancing integrin signaling. Cell. 2009;139(5):891-906.

21. Atsawasuwan P, Mochida Y, Katafuchi M, Kaku M, Fong KS, Csiszar K, Yamauchi M. Lysyl oxidase binds transforming growth factor-beta and regulates its signaling via amine oxidase activity. J Biol Chem. 2008; 283(49):34229-240

22. Kim YM, Kim EC, Kim Y. The human lysyl oxidase-like 2 protein functions as an amine oxidase toward collagen and elastin. Mol Biol Rep. 2011; 38(1):145-49.

23. Peyrol S, Raccurt M, Gerard F, Gleyzal C, Grimaud JA, Sommer P. Lysyl oxidase gene expression in the stromal reaction to in situ and invasive ductal breast carcinoma. Am J Pathol. 1997;150(2):497-507.

24. Santhanam AN, Baker AR, Hegamyer G, Kirschmann DA, Colburn NH. $\mathrm{Pdcd} 4$ repression of lysyl oxidase inhibits hypoxia-induced breast cancer cell invasion. Oncogene. 2010;29(27):3921-32.

25. Paszek MJ, Weaver VM. The tension mounts: mechanics meets morphogenesis and malignancy. J Mammary Gland Biol Neoplasia. 2004;9(4):325-42.

26. Paszek MJ, Zahir N, Johnson KR, Lakins JN, Rozenberg GI, Gefen A, Reinhart-King CA, Margulies SS, Dembo M, Boettiger D, Hammer DA, Weaver VM. Tensional homeostasis and the malignant phenotype. Cancer Cell. 2005;8(3):241-54.

27. Wozniak MA, Desai R, Solski PA, Der CI, Keely PJ. ROCK-generated contractility regulates breast epithelial cell differentiation in response to the physical properties of a three-dimensional collagen matrix. J Cell Biol. 2003;163(3):583-95.

28. Chun TH, Hotary KB, Sabeh F, Saltiel AR, Allen ED, Weiss SJ. A pericellular collagenase directs the 3-dimensional development of white adipose tissue. Cell. 2006;125(3):577-91.

29. Oi C, Wei B, Zhou W, Yang Y, Li B, Guo S, Li J, Ye J, Li J, Zhang O, Lan T, He $X$, Cao L, Zhou J, Geng J, Wang L. P-selectin-mediated platelet adhesion promotes tumor growth. Oncotarget. 2015;6(9):6584-96. 